\title{
RECIST Partial Response
}

National Cancer Institute

\section{Source}

National Cancer Institute. RECIST Partial Response. NCI Thesaurus. Code C159547.

At least a 30\% decrease in the sum of the longest diameter of target lesions, taking as reference the baseline sum longest diameter. 